\title{
Photophysics and Photonics of Heteroepitaxial Organic Nanofibers
}

\author{
Francesco Quochi, Michele Saba, Andrea Mura and Giovanni Bongiovanni \\ Dipartimento di Fisica, Università di Cagliari, \\ Italy
}

\section{Introduction}

Organic epitaxy is a powerful technique to grow highly ordered organic aggregates at crystalline surfaces. Long-range epitaxial order could be successfully exploited to enhance optoelectronic properties of organic molecular thin films for device applications. A relevant example of organic epitaxy on inorganic substrates is represented by high-vacuum deposition of para-sexiphenyl (p-6P) on (001)-oriented muscovite mica (Simbrunner et al., 2011), which yields large domains of parallel and crystalline nanofibers with extremely high surface-tovolume ratios, sub-wavelength cross-sectional dimensions, and lengths up to the millimeter scale (Yanagi et al., 1999; Andreev et al., 2000; Balzer \& Rubahn, 2001). These nanofibers exhibit excellent photonic and optoelectronic properties: high blue photoluminescence efficiency (Stampfl et al., 1995), high in-fiber carrier mobility (Birendra-Singh et al., 2006; Kjelstrup-Hansen et al., 2006), luminescence guidance (Balzer et al., 2003), in-fiber amplification of spontaneous emission with large gain factors (Quochi et al., 2006; Cordella et al., 2007), and optically-induced laser action (Quochi et al., 2004, 2005, 2008). Very recently, organic-organic heteroepitaxy of mixed $p$-6P/alpha-sexithiophene (6T) nanofibers with highly polarized blue, green and red emission has been successfully demonstrated (Simbrunner et al., 2010), further broadening the range of potential applications of organic epitaxial nanofibers in photonics and optoelectronics.

In this paper, we will review the optical and photonic properties of $p-6 \mathrm{P}$ and mixed $6 \mathrm{~T} / p-6 \mathrm{P}$ nanofibers epitaxially grown on oriented muscovite mica by hot-wall epitaxy and organic molecular beam epitaxy. We will first present ensemble studies of the amplified spontaneous emission and lasing action properties of nanofibers. The results of combined optical and morphological studies on individual nanofibers will be reported and the waveguide optical gain and lasing threshold figures will be given. We will then discuss the excited-state dynamics of nanofibers, as well as the intrinsic stimulated-emission perfomance of nanofibers in terms of gain lifetime and bandwidth. Potential applications of organic epitaxial nanofibers in photonics and sensing technologies will also be touched upon. Last, we will present recent results obtained in mixed $6 \mathrm{~T} / p$-6P nanofibers displaying high degree of epitaxial alignment, and highly polarized and broadband emission extending from the blue to the red.

\section{Growth and characterization of $p-6 \mathrm{P}$ epitaxial nanofibers}

Para-sexiphenyl nanofiber films are grown on freshly-cleaved, (001)-oriented muscovite mica by Hot-Wall Epitaxy (Andreev et al., 2000) and Organic Molecular Beam Epitaxy 
(Balzer \& Rubahn, 2011). In the hot-wall epitaxy technique, $p$-6P is purified by threefold sublimation under dynamic vacuum. The system is operated with a nominal base pressure during growth of about $9 \times 10^{-6}$ mbar and the $p$ - $6 \mathrm{P}$ source temperature is set to the optimized value of $240{ }^{\circ} \mathrm{C}$. The substrate temperature is varied between 90 and $180^{\circ} \mathrm{C}$, while the growth time is varied between $10 \mathrm{~s}$ and $120 \mathrm{~min}$. Further details can be found in (Plank et al., 2001; Andreev et al., 2001). In the organic molecular beam epitaxy technique, sheets of muscovite mica are cleaved in air and are transferred immediately after cleavage into a high-vacuum apparatus (base pressure of $5 \times 10^{-8} \mathrm{mbar}$ ). Before organic material is deposited, the samples are outgassed at a temperature of around $130{ }^{\circ} \mathrm{C}$ such that low energy electron diffraction shows the well-known hexagonal surface structure of clean mica with electric surface dipoles present. Para-sexiphenyl is deposited from a home-built Knudsen cell by vacuum sublimation; during the deposition the pressure inside the vacuum system rises to $2 \times 10^{-7}$ mbar. Long $p$-6P needles grow for deposition rates of $0.1 \AA / s$ and at substrate temperatures around $150{ }^{\circ} \mathrm{C}$.

Morphological characterization of the nanofibers is performed by scanning-probe atomic force microscopy using Si-tip probes in tapping mode in air. Complementary optical characterization is carried out by $\mathrm{cw}$ epifluorescence measurements using an inverted microscope with a $\mathrm{Hg}$ high-pressure lamp as the excitation source.

\subsection{Dependence of nanofiber morphology on deposition time}

Topography studies of the surface morphology of films realized by hot-wall epitaxy at $130^{\circ} \mathrm{C}$ substrate temperature with different growth times demonstrate that oriented and mutually parallel nanofibers are formed by progressive regrouping of individual crystallites originating at the early growth stages for deposition times $<10-25 \mathrm{~s}$ (Andreev et al., 2006). For growth times longer than $5 \mathrm{~min}$., only linear fibers are observed. Surface topographic images of films obtained with various growth times are shown in Fig. 1. For long (>40 min.) deposition times, close-packed and interconnected nanofibers are realized.

X-ray diffraction studies demonstrated that $p$-6P nanocrystals packed in nanofibers are cooriented and characterized by well-defined epitaxial relationships to the mica substrate: the long molecular axis of $p-6 \mathrm{P}$ is nearly parallel to the mica surface plane and nearly pependicular to the fiber axes (Plank et al., 2003). Long-range azimuthal order is realized, which results in high optical anisotropy and high carrier mobility (Andreev et al., 2000; Birendra Singh et al., 2006).

Statistical analysis of topographic images reveal that the morphological parameters of nanofibers are strongly correlated to the growth time. Raising growth time from 40 to 120 min. results in an increase of fiber mean height from $\sim 110$ to $\sim 290 \mathrm{~nm}$, whereas the mean base width increases from $\sim 210$ to $\sim 350 \mathrm{~nm}$. Deposition time turns out to be a knob to tune cross-sectional dimensions of nanofibers; cross-sectional size is crucial to the attainment of optical waveguiding and amplificaton in nanofibers.

Closer inspection to the topographic images shows that nanofibers are characterized by the presence of breaks, resulting in fiber segmentation (clearly visible in the images shown in Fig. 2). Such breaks, $\sim 50$ to $\sim 300 \mathrm{~nm}$ in width, occur at the end of the material growth process as a possible result of a surface thermal gradient during substrate cooling (Balzer \& Rubahn, 2001). Breaks play a crucial role for the active photonic properties of nanofibers since they are responsible for the establishment of optical feedback along the nanofiber's axes and for light scattering into out-of-plane directions (see Sec. 3.2). 

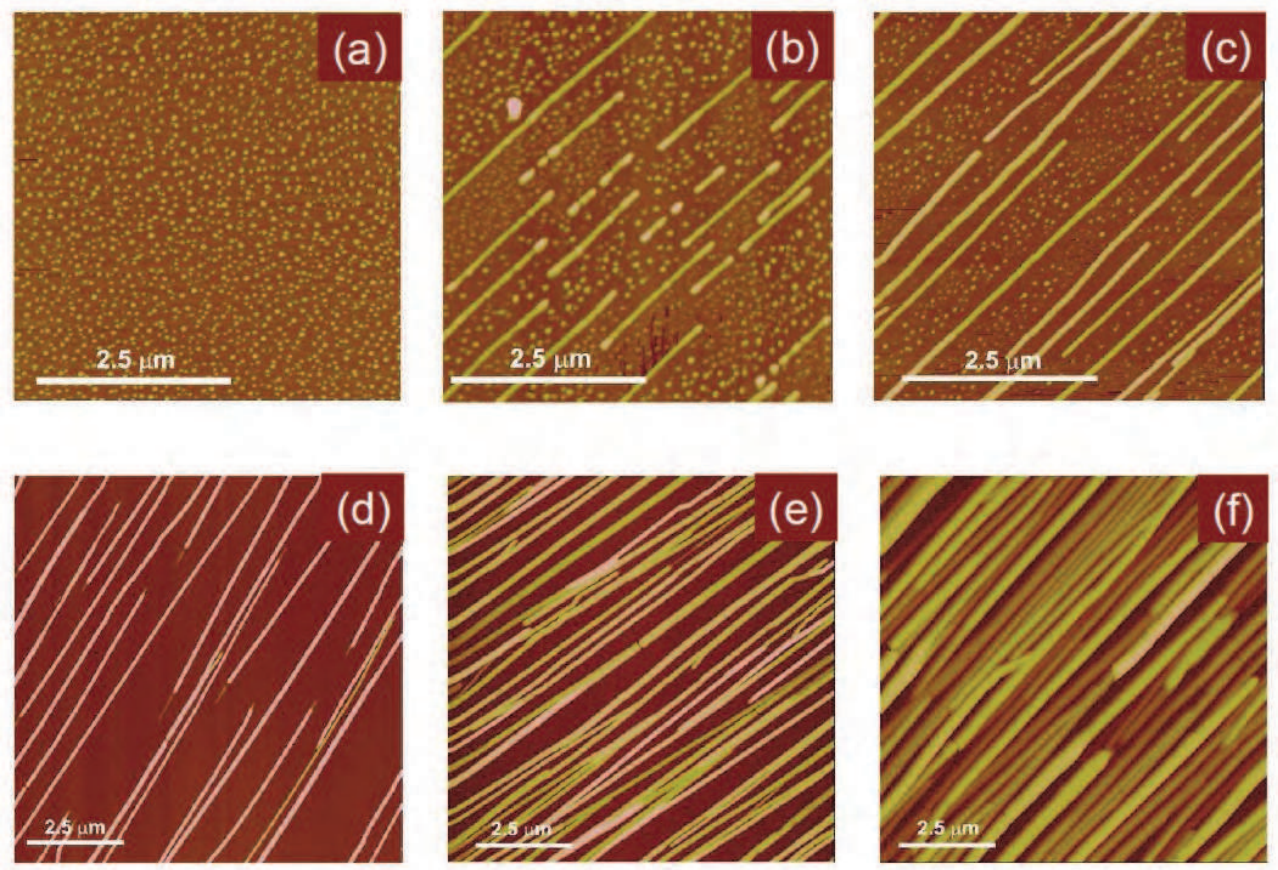

Fig. 1. AFM topography images of $p-6 \mathrm{P}$ nanofiber films grown by hot-wall epitaxy on (001)oriented muscovite mica with (a) $10 \mathrm{~s}$, (b) $25 \mathrm{~s}$, (c) $90 \mathrm{~s}$, (d) 5 min., (e) 40 min., and (f) 120 min. deposition time. The growth temperature is $130^{\circ} \mathrm{C}$. The height scale is $0-50 \mathrm{~nm}$ in (a) (c), $0-100 \mathrm{~nm}$ in (d), 0-220 $\mathrm{nm}$ in (e), and $0-700 \mathrm{~nm}$ in (f).

\subsection{Dependence of nanofiber morphology on substrate temperature}

Substrate temperature is also an important growth parameter for tuning the morphological properties of nanofibers. In Fig. 2 are displayed topographic images of nanofiber films grown for $60 \mathrm{~min}$. by hot-wall epitaxy at different substrate temperatures. As temperature is raised from 90 to $170^{\circ} \mathrm{C}$, mean fiber height and cross-sectional area increase from $\sim 80$ to $\sim 300 \mathrm{~nm}$ and from $\sim 0.05$ to $\sim 0.3 \mu \mathrm{m}^{2}$, respectively, whereas the surface coverage (percentage of film surface covered by nanofibers) decreases from 70 to $20 \%$, approximately. These behaviors are consistent with Arrhenius dependences, that is, nanofiber nucleation is a thermally activated process (Kankate et al., 2008). Activation energies range from a few tens to hundreds of meV, depending on whether hot-wall or beam deposition is used and on the morphological parameter being analyzed.

Strong correlation is found between fiber morphology and substrate temperature during growth. Hence, substrate temperature can also be used to control the photonic properties of nanofibers. 

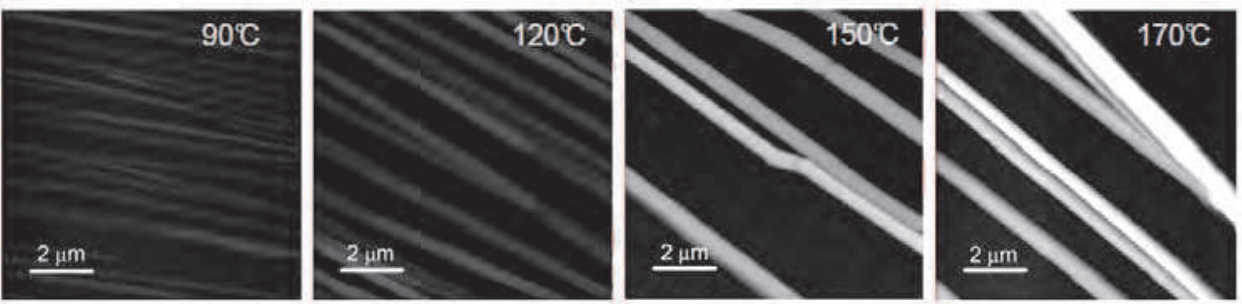

Fig. 2. Topographic images of the surface morphology of $p-6 \mathrm{P}$ nanofiber films grown by hotwall epitaxy on (001)-oriented muscovite mica at different substrate temperatures and with $60 \mathrm{~min}$. deposition time. The height scale is $0-500 \mathrm{~nm}$.

\subsection{Epifluorescence microspectroscopy of nanofibers}

Epifluorescence microscopy and microspectroscopy characterization techniques provide valuable information on the emission properties of nanofiber films. The inset of Fig. 3 shows an epifluorescence micrograph of an ensemble of nanofibers under UV illumination.

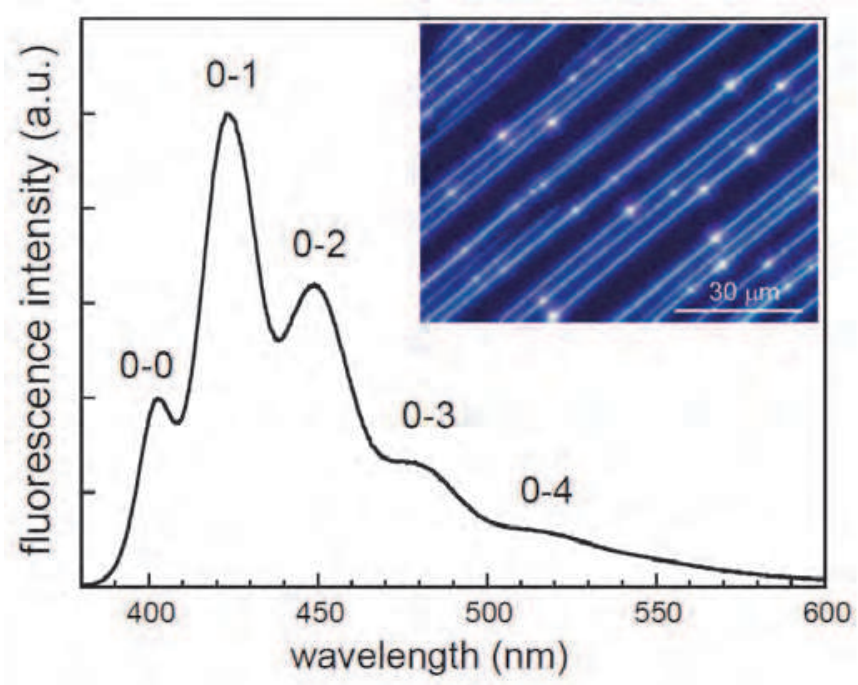

Fig. 3. Epifluorescence spectrum (main panel) and micrograph (inset) of an ensemble of $p-6 \mathrm{P}$ nanofibers photoexcited by a Hg high-pressure lamp spectrally narrowed in the $330-360 \mathrm{~nm}$ band. Emission bands originating from vibronic coupling with $\mathrm{C}-\mathrm{C}$ stretching mode are labeled for clarity.

Large enhancement of fluorescence scattering into out-of-plane directions is found to occur in correspondence of fiber breaks. These bright spots provide high sensitivity detection of $p$ $6 \mathrm{P}$ epifluorescence. The main panel of Fig. 3 displays the room-temperature epifluorescence spectrum of photoexcited $p$-6P epitaxial nanofibers. The spectrum shows the typical features of an H-type molecular aggregate, that is, a weak electronic (0-0) transition and a more intense vibronic progression involving the $\mathrm{C}-\mathrm{C}$ stretching mode. 


\section{Optical amplification and lasing action in $p-6 \mathrm{P}$ epitaxial nanofibers}

Nonlinear emission properties of $p-6 \mathrm{P}$ epitaxial nanofibers are investigated by transient fluorescence spectroscopy using ultrashort ( 150 fs) laser pulses delivered by a frequencydoubled ( $\sim 390 \mathrm{~nm}$ wavelength) Ti:sapphire amplifield laser running at a repetition frequency of $1 \mathrm{kHz}$. The pump laser beam is focused to circular spots ranging from 120 to $180 \mu \mathrm{m}$ in diameter on the nanofiber films, allowing to excite simultaneously tens of nanofibers with pulsed fluences up to $1 \mathrm{~mJ} / \mathrm{cm}^{2}$. For nonlinear emission experiments, the pump field polarization is set perpendicular to the axis of the nanofibers (thus parallel to the long axis of the $p$-6P molecules) for maximum optical absorption and the excitation fluence is varied using a variable density filter. In polarization-resolved experiments, the polarization of the optical emission is rotated using a retardation plate. The optical emission is dispersed in a single grating spectrometer equipped with a liquid- $\mathrm{N}_{2}$-cooled chargecoupled device allowing for high-sensitivity, time-integrated measurements.

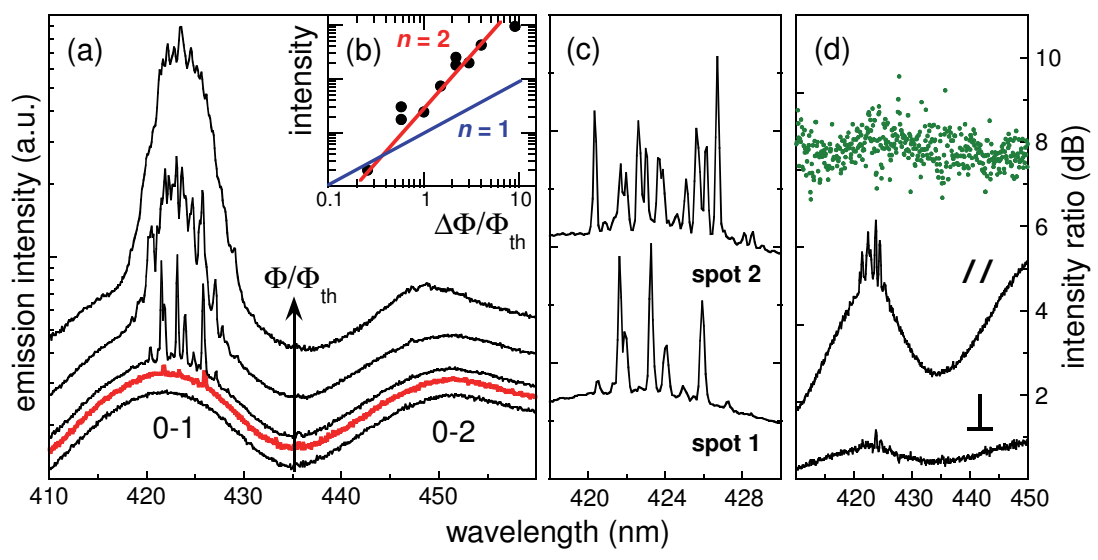

Fig. 4. (a) Time-integrated optical emission spectra of $p-6 \mathrm{P}$ epitaxial nanofibers excited by subpicosecond pulses as a function of pump fluence: $0.8,1.0,1.6,3.0,10$. Relative units refer to the $\Phi / \Phi_{\text {th }}$ ratio, where $\Phi_{\text {th }}$ is lasing threshold fluence. (b) Time- and spectrally-integrated intensity of nonlinear emission above lasing threshold. Linear and quadratic dependences are shown by the red and blue line, respectively. (c) Position sensitivity of lasing modes: emission spectra are taken in different positions of the pump laser spot on the film surface. (d) Polarization-resolved emission spectra, acquired upon polarization filtering of the emission along the direction parallel $(/ /)$ and perpendicular $(\perp)$ to the long molecular axis of $p-6 \mathrm{P}$. Spectral dependence of intensity ratio is displayed as green dots.

The evolution from spontaneous emission to coherent (lasing) emission for increasing pump pulsed fluence is shown in Fig. 4(a). The emission spectrum relates to tens of interconnected nanofibers and thus represents an ensemble-averaged response of nanofibers. The films are kept at room temperature. When the pump fluence exceeds a threshold value $\Phi_{\text {th }}$, resolution-limited peaks emerge from the spontaneous emission spectrum on the $0-1$ vibronic band. Threshold fluences are position dependent and can vary from a few $\mu \mathrm{J} / \mathrm{cm}^{2}$ to hundreds of $\mu \mathrm{J} / \mathrm{cm}^{2}$. As pump fluence is much increased above 
threshold, the visibility of the narrow lines decreases until spectral narrowing of the vibronic peak dominates the system response. The narrow peaks denote the presence of coherent optical feedback within the $p$-6P nanofiber films, whereas spectral narrowing of the $0-1$ and $0-2$ emission peaks indicates that amplified spontaneaous emission occurs at the high pump levels. In Fig. 4(b), the emission intensity is plotted as a function of the normalized pump excess fluence, defined as $\Delta \Phi / \Phi_{\text {th }}=\left(\Phi-\Phi_{\text {th }}\right) / \Phi_{\text {th }}$. The signal intensity is spectrally integrated over the 0-1 band upon subtraction of the spontaneous emission contribution. The nonlinear dependence of the emission intensity on $\Delta \Phi / \Phi_{\text {th }}$ (slope efficiency that increases with increasing pump fluence) is partially attributed to the fact that the number of coherent modes reaching oscillation theshold increases with raising pump excess fluence (Quochi et al., 2004).

The spatial sensitivity of nolinear lasing in $p-6 \mathrm{P}$ crystalline nanofibers are reported in Fig. 4(c). Different distributions of unequally spaced modes are realized in different positions of the excitation spot on the film surface, suggesting the occurrence of coherent random lasing (Cao, 2003, 2005). Moving the pump spots across the fim surface results in the excitaton coherent modes having different resonance wavelengths and optical losses; as a consequence, strong variation in lasing threshold fluence is registered as the excited area is changed. As expected from the film structural anisotropy and epitaxial alignment, both linear and nonlinear optical emissions exhibit strong polarization anisotropy with intensity ratios as large as $10 \mathrm{~dB}$ (Fig. $4(\mathrm{~d})$ ).

Detailed studies on optical amplification and random lasing action are conducted on singly selected, isolated $p$-6P epitaxial nanofibers.

\subsection{Guided amplification of spontaneous emission}

Determination of net optical gain in isolated fibers yields the optical amplification performance of $p$-6P epitaxial nanofiber waveguides. The experiments are performed on nanofibers not showing the presence of strong scattering centers and displaying fairly uniform epifluorescence efficiency (Quochi et al., 2006).

Singly selected fibers grown by organic molecular beam epitaxy and excited by 150 fs-long pulses with $390 \mathrm{~nm}$ central wavelength are investigated by optical microspectroscopy. The optical emission, excited through the back (substrate) surface, is collected from the front surface using a microscope objective and focused onto the input slit of the spectrometer. Setting the spectrometer to zeroth-order diffraction and fully opening the input slit, fluorescing fibers covering the photoexcited area can be imaged with $\sim 2 \mu \mathrm{m}$ spatial resolution. Tuning the spectrometer to first-order diffraction and narrowing the input slit, the emission of individual nanofibers aligned parallel to the input slit can be spectrally resolved. Results obtained on a $\sim 40 \mu \mathrm{m}$-long nanofiber are shown in Fig. 5. At low excitation levels, the emission micrograph of a selected nanofiber shows uniform emission intensity between the fiber tips (Fig. 5(a)). Increasing the pump fluence above a threshold value of $\sim 100 \mu \mathrm{J} / \mathrm{cm}^{2}$ per pulse, enhancement of the emission intensity is detected near the fiber tips (Fig. 5(b)). The emission intensity increases continuously as the position approaches the tips, where the guided light is efficiently outcoupled (Fig. 5(c)).

Assuming linear amplification of spontaneous emission and uniform scattering efficiency across the fiber, the emission intensity profile can be fitted by the function $I_{\mathrm{T}}(z)=I(z)+I(L-z)$, where $I(z) \sim[\exp (g z)-1] / g ; z$ is the distance from a fiber tip, $g$ the net modal gain coefficient, and $L$ the fiber length. 


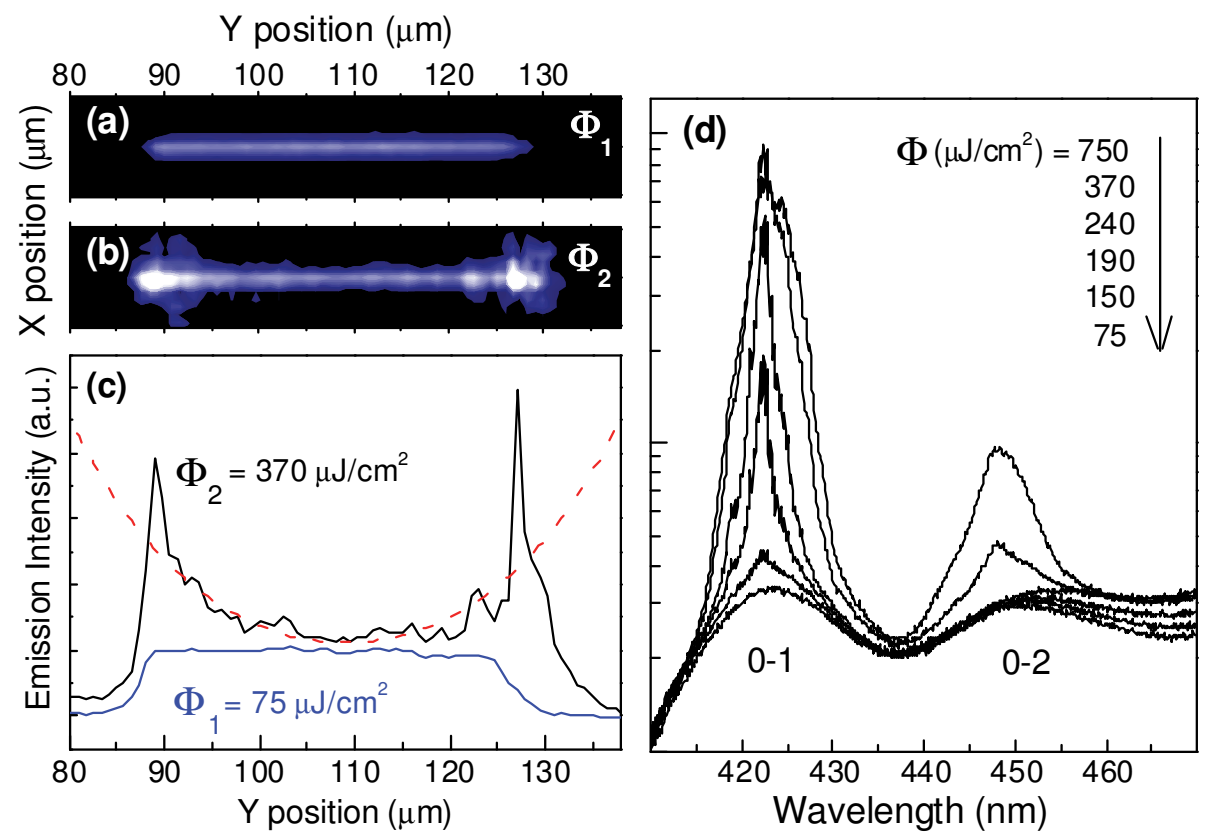

Fig. 5. (a,b) Time-integrated emission micrographs of an individual nanofiber excited by ultrafast laser pulses, for a pump pulsed fluence $\Phi_{1}=75$ (a) and $\Phi_{2}=370 \mu \mathrm{J} / \mathrm{cm}^{2}$. (c) Spatial profiles of the emission intensity. The dashed line is the fit of a linear amplification function (see text) to the measured profile. (d) Emission spectra for different values of the excitation fluence. The spectra are spatially integrated over the nanofiber region.

The occurrence of amplified spontaneous emission is confirmed by the spectral analysis of the emission intensity above amplification threshold, which shows line narrowing of the emission towards the center of the vibronic bands (Fig 5(d)), whereas spectral fringes relating to $\sim 40 \mu \mathrm{m}$-long Fabry-Perot resonator are not visible. Curve fitting of intensity profiles yields $g_{1} \sim 1200 \mathrm{~cm}^{-1}$ for the $0-1$ emission band and $g_{2} \sim 700 \mathrm{~cm}^{-1}$ for the $0-2$ emission band at the highest excitation fluence of $750 \mu \mathrm{J} / \mathrm{cm}^{2}$. Overall, the results demonstrate that $p-$ $6 \mathrm{P}$ epitaxial nanofibers are able to guide and amplify their spontaneous emission with large amplification factors, and thus hold potential as active nanoamplifiers at surfaces.

\subsection{Random lasing action}

Optical studies on individual $p$-6P nanofibers with strong scattering centers allow us to understand the origin of coherent optical feedback, which is responsible for random lasing action. Isolated nanofibers give the opportunity to study one-dimensional random lasing in self-assembled epitaxial nanostructures. To this aim, we apply the same microspectrographic technique used to characterize amplified spontaneous emission in homogeneous, break-free fibers. Emission spectra of isolated nanofibers grown by organic molecular beam epitaxy show that random lasing threshold is reached at both the 0-1 and 02 vibronic peaks. As expected for a single laser emitter, spontaneous emission saturates at 
the value reached at threshold (Quochi et al., 2006). On the contrary, fluorescence clamping is not observed in ensemble-averaged measurements, since not all the excited fibers reach lasing threshold (Fig. 4).

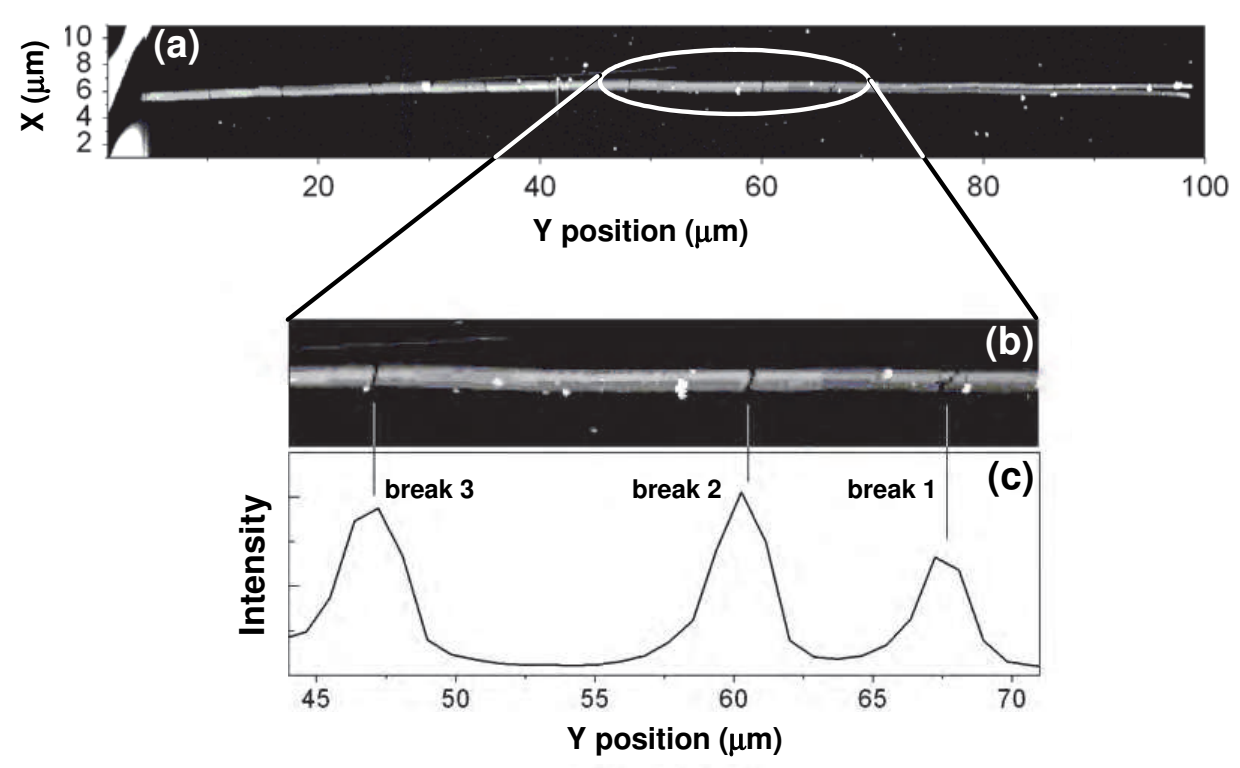

Fig. 6. (a) Topographic image of a segmented $p$-6P epitaxial nanofiber. (b) Image of the indicated subsection of the nanofiber. (c) Lasing intensity profile relating to the same fiber segment as in (b). Vertical marks highlight the correspondence between the positions of breaks and those of optical scattering centers responsible for coherent feedback and laser light outcoupling towards collecting optics.

Correlated lasing measurements and atomic-force topographic measurements give insight into the origin of one-dimensional coherent feedback in individual $p$-6P nanofibers. Fig. 6(a) shows the topographic image of a $\sim 100-\mu \mathrm{m}-\mathrm{long}$, selected nanofiber. When the fiber morphology is compared to the lasing intensity profile on the same region (Figs. 6(b) and 6(c)), it clearly turns out that scattering of the guided lasing emission occurs at the fiber breaks. In fact, excellent correspondence is found between the positions of the bright lasing spots and those of the fiber breaks. These findings strongly suggest that back-reflections of the fiber waveguide modes at the fiber break interfaces are the main source of optical feedback along the nanofibers' axes. Our interpretation of the experimental data in terms of one-dimensional coherent random lasing is in fact supported by calculations of the resonant optical modes of one-dimensionally disordered systems (Quochi et al., 2005).

Substrate temperature used during growth has a big influence on the lasing threshold performance of $p$-6P epitaxial nanofibers. Increasing substrate temperature from 90 to $170^{\circ} \mathrm{C}$, mean lasing threshold, as measured in films grown by hot-wall epitaxy with $60 \mathrm{~min}$. deposition time, decreases from $\sim 300$ to $\sim 50 \mu \mathrm{J} / \mathrm{cm}^{2}$. These findings are traced back to the 
thermally activated growth of $p$-6P epitaxial nanofibers; in fact, thermal activation of the cross-sectional size of nanofibers should result in strong temperature increase of the optical confinement factor of the guided optical emission. Therefore, substrate temperature can also be used to tune the active photonic properties of $p$-6P epitaxial nanofibers.

\section{Excited-state dynamics and photophysics of $p-6 \mathrm{P}$ epitaxial nanofibers}

The potential of organic films for laser device technologies stems from the ability to achieve lasing thresholds compatible with indirect electrical pumping by unexpensive and convenient light sources. Lasing performance results from both extrinsic factors (i.e., optical confinement of lasing modes in the gain medium, propagation and feedback losses), and intrinsic gain performance of the active medium. The latter is quantified by the figure of merit for optical amplification, which can be defined as $F O M=\sigma_{\mathrm{G}} B_{\mathrm{G}} \tau$, where $\sigma_{\mathrm{G}}$ is the netgain cross-section, $B_{\mathrm{G}}$ its bandwidth, and $\tau$ the excited-state lifetime at operating excitation intensity. These parameters can be retrieved from the analysis of the excited-state dynamics of the system.

We resort to time-resolved fluorescence and transient differential absorption measurements with ultrafast excitation to study the excited-state dynamics of $p$-6P epitaxial nanofiber films. We investigate ensembles of close-placked nanofibers displaying high amplification and lasing thresholds $\left(>100 \mu \mathrm{J} / \mathrm{cm}^{2}\right)$ to ensure that stimulated emission affects population dynamics only weakly. Ultrafast excitation experiments are performed using 150-fs-long pulses delivered by an optical parametric amplifier allowing to tune the excitation wavelength across the 350-400 nm interval. The sample emission is dispersed in a single grating spectrometer and temporally resolved by a visible streak camera with $\sim 20$ ps time resolution. A cold-finger cryostat fed with liquid air is used to vary the sample temperature in the 80-300 $\mathrm{K}$ range. Complementary differential transmission $(\Delta T / T)$ measurements are performed using broadband pulses obtained by supercontinuum generation in a sapphire plate as the optical probe. Time delay betwen the pump and probe pulses is controlled by a motorized optical delay stage.

\subsection{Ultrafast time-resolved photoluminescence spectroscopy}

Main results of time-resolved fluorescence studies are reported in Fig. 7, where fluorescence decay rate constant is plotted as a function of the pump pulsed fluence. Fluorescence decay traces taken at $80 \mathrm{~K}$ are drawn in the inset of Fig. 7 (Quochi et al., 2008).

At high pump fluences, the excited-state decay dynamics is dominated by nonradiative, density-dependent processes ascribed to singlet-singlet (bimolecular) annihilations. Bimolecular recombination strongly depends on lattice temperature. Upon fitting the initial $1 / e$ decay times with the results of model simulations of the decay dynamics, we estimate that the bimolecular coefficient $\left(\kappa_{\mathrm{SS}}\right)$ increases from $3 \times 10^{-9}$ to $4 \times 10^{-8} \mathrm{~cm}^{3} / \mathrm{s}$ when lattice temperature is increased from 80 to $300 \mathrm{~K}$. Linear (monomolecular) recombination rate constant $\left(k_{0}\right)$ also exhibits a temperature dependence, concomitanly increasing from $1 \times 10^{9}$ to $2.3 \times 10^{9} \mathrm{~s}^{-1}$. At $80 \mathrm{~K}$, bimolecular processes kick in for pump fluences of $\sim 2-4 \mu \mathrm{J} / \mathrm{cm}^{2}$, whereas the threshold goes down to $0.1 \mu \mathrm{J} / \mathrm{cm}^{2}$ at room temperature. Lattice temperature dependence of bimolecular processes suggests that exciton migration is a thermally activated process (Wiesenhofer et al., 2006). Activation energy is possibly related to the presence of energetic disorder in $p$-6P nanostructured films (Kadashchuk et al., 2004). 


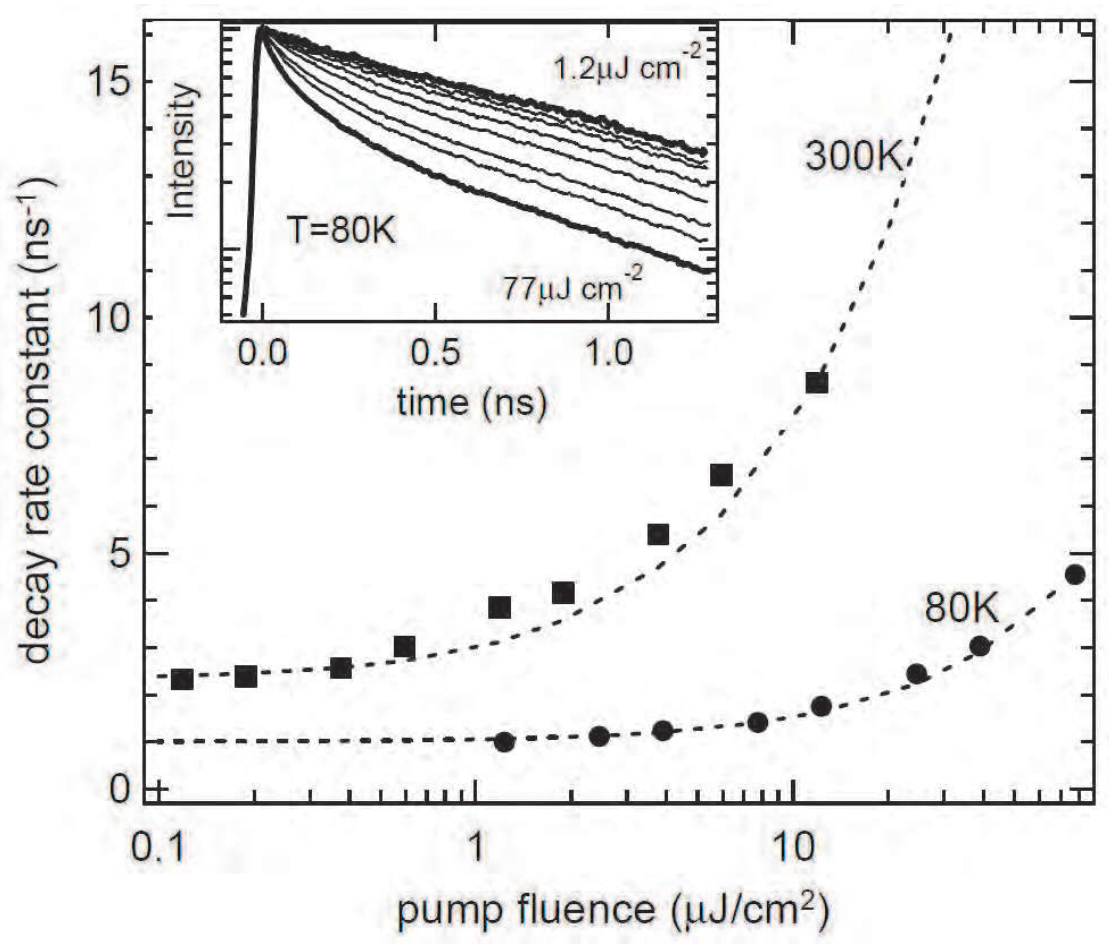

Fig. 7. Fluorescence decay rate constant of $p$-6P nanofibers excited by ultrafast pulses at 380 $\mathrm{nm}$, at $300 \mathrm{~K}$ (squares) and $80 \mathrm{~K}$ (circles). Dashed lines: Calculated decay rate constant; the calculation includes both monomolecular and bimolecular recombination processes. Inset: Fluorescence decay traces measured at $80 \mathrm{~K}$ with pump pulsed fluence of 1.2, 2.5, 3.9, 7.8, 12, 25,39 , and $77 \mu \mathrm{J} / \mathrm{cm}^{2}$.

\subsection{Ultrafast transient absorption spectroscopy}

Excited-state photophysical processes such as stimulated emission and photoinduced absorption are studied by differential transmission measurements as a function of pumpprobe time delay. Transmission spectra taken for various pump-probe delays are shown in Fig. 8. At short delays ( $\leq 1 \mathrm{ps})$, the system response is characterized by broadband stimulated emission extending from the deep blue to the orange $\left(B_{\mathrm{G}} \sim 1 \mathrm{eV}\right)$ and exhibiting the vibronic progression of singlet excitons in $p-6 \mathrm{P}$ crystalline nanofibers. The stimulatedemission cross-section is found to be $\sim 2 \times 10^{-16} \mathrm{~cm}^{2}$, as estimated from $\Delta T / T$ signal amplitude at the 0-2 vibronic peak at zero time delay, assuming that primary photoexcitations are singlet excitons. On the long wavelength side, the spectrum is dominated by photoinduced absorption of triplet excitons and polarons promptly excited by the pump pulses. At room temperature and high pump fluences $\left(\sim 90 \mu \mathrm{J} / \mathrm{cm}^{2}\right)$, broadband stimulated emission decays rapidly due to singlet-singlet annihilations and a photoinduced absorption band arises near the optical gap of the material at $\sim 400 \mathrm{~nm}$. 


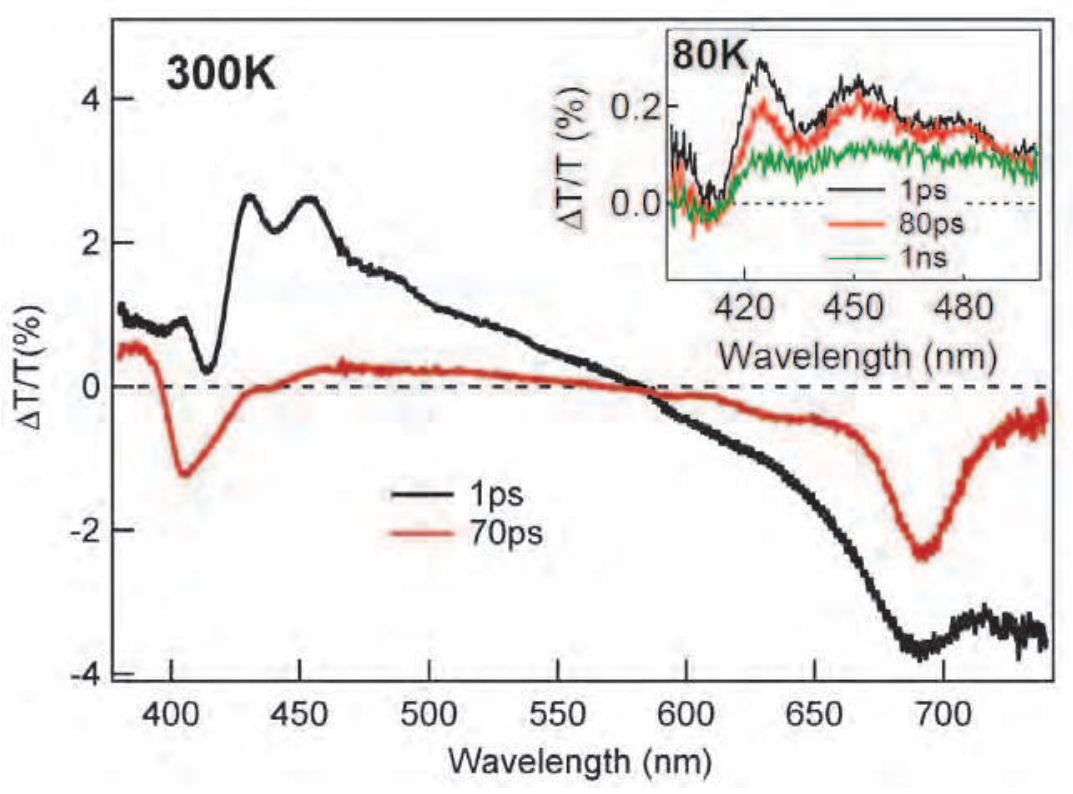

Fig. 8. Differential transmission spectra of $p$-6P epitaxial nanofibers excited by ultrafast pulses centered at $360 \mathrm{~nm}$, for different lattice temperatures and pump-probe time delays. Pump pulsed fluence: $90 \mu \mathrm{J} / \mathrm{cm}^{2}$ (main panel); $10 \mu \mathrm{J} / \mathrm{cm}^{2}$ (inset).

This long-lived photoinduced absorption band (decay time $>1 \mathrm{~ns}$ ) is attributed to intermolecular excitons with charge-transfer character and generated via singlet-singlet annihilations (Cordella et al., 2007). At cryogenic temperatures $(80 \mathrm{~K})$ and lower excitation fluences $\left(\sim 10 \mu \mathrm{J} / \mathrm{cm}^{2}\right)$, bimolecular recombination is strongly suppressed: excited-state lifetime $(\tau)$ increases up to $\sim 1$ ns and the photoinduced absorption band associated to secondary charge-transfer excitons disappears (inset of Fig. 8).

Excited-state lifetime shortening is the primary effect of singlet-singlet annihilations. As a secondary effect, a (nonlinear) population of intermolecular excitons is created, whose absorption spectrum overlaps with gain spectrum of emissive (singlet) excitons. Combined effects of lifetime shortening, net-gain reduction and bandwidth shrinking by photoinduced absorption are detrimental to lasing action in nanofibers excited by long (nanosecond) pulses.

\subsection{Monomolecular lasing}

Sample cooling to cryogenic temperatures makes it possible to circumvent fast bimolecular decay and photoinduced absoption by intermolecular excitons. Direct demonstration of occurrence of monomolecular lasing, that is, lasing in the linear recombination regime at cryogenic temperatures is provided in Fig. 9, which reports the time-wavelength spectrogram of the emission intensity of nanofibers excited by subpicosecond pulses. 


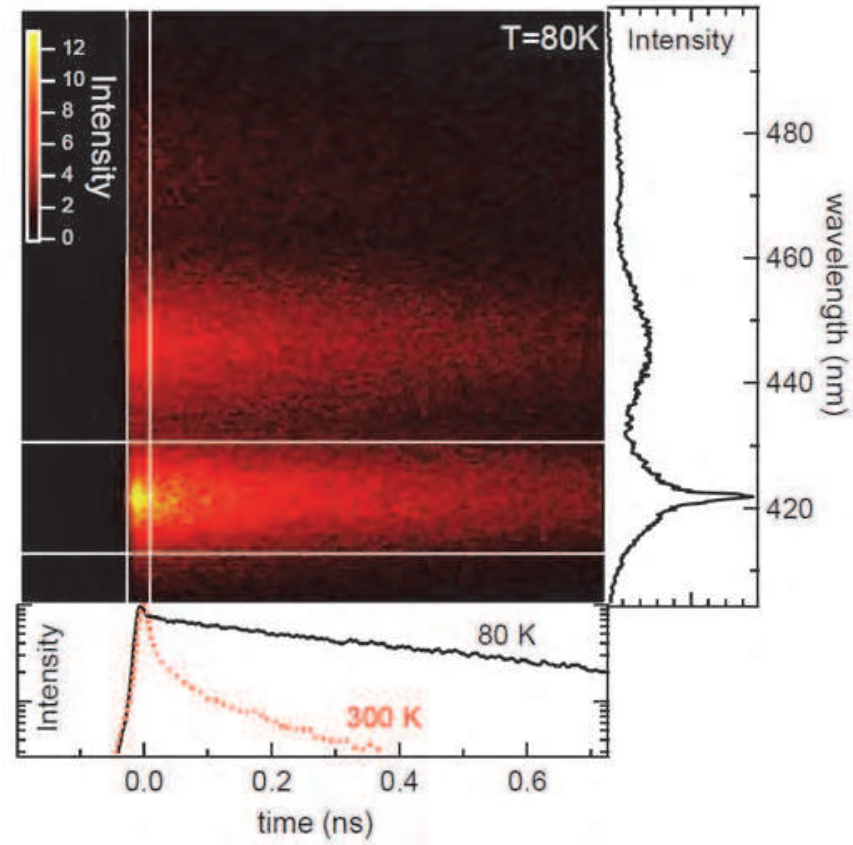

Fig. 9. Time-wavelength spectrogram of the fluorescence intensity of cryogenically cooled nanofibers, excited by subpicosecond pulses with $360 \mathrm{~nm}$ central wavelength. Colors code for intensity levels on an arbitrary unit scale. Pump pulsed fluence is $7 \mu \mathrm{J} / \mathrm{cm}^{2}$. Vertical (horizontal) white lines delimit the spectrogram region used for temporal (spectral) integration. Right panel: emission spectrum integrated in the first 30 ps after excitation pulse arrival. Bottom panel: Emission decay traces integrated over the $0-1$ vibronic band of the $p$ $6 \mathrm{P}$ emission. The solid (dashed) line depicts the time profile at $80(300) \mathrm{K}$.

The spectral profile (right panel) shows evidence of lasing emission on top of the 0-1 spontaneous emission band. The time profile analysis (bottom panel) demonstrates that at $80 \mathrm{~K}$ prompt laser emission decays rapidly, leaving the system with a population of singlet excitons undergoing monomolecular recombination with $\sim 1$ ns decay time (Quochi et al., 2008).

\section{Para-sexiphenyl epitaxial nanofibers for active photonic sensing}

Low-threshold random lasing action could be exploited to achieve high photonic sensitivity to various agents; miniaturized random laser sources are thus be envisaged to enable new functionalities for next-generation technologies (Wiersma, 2000; Wiersma \& Cavalieri, 2001). Based on model simulations of the coherent optical response of a random medium (Quochi et al., 2005), we estimate that a typical $p$-6P epitaxial nanofiber with a length of $\sim 100 \mu \mathrm{m}$ and a dozen of thin $(\sim 200 \mathrm{~nm})$ breaks would display attoliter sensitivity to contamination by index matching fluids (Fig. 10(a)). Also, strain sensors with very large $\left(>10^{3}\right)$ gauge factor and high dynamic range could be obtained by optical interrogation of single nanofibers aligned parallel to the strain axis (Figs. 10(b) and 10(c)). 


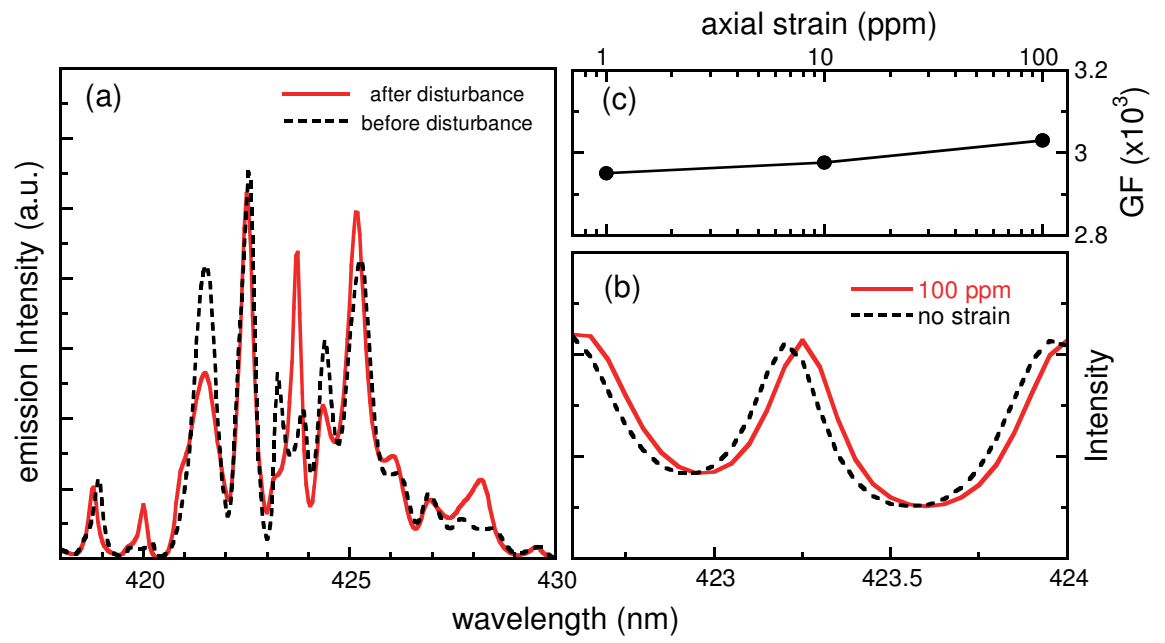

Fig. 10. Model calculations of the photonic sensitivity of a $p$-6P epitaxial nanofiber with onedimensional random optical feedback. (a) Coherent emission spectrum before and after optical neutralization of a fiber break upon air-gap filling with index matching fluid. (b) Resonance wavelength shift in response to $100 \mathrm{ppm}$ axial strain. (c) Strain gauge factor as a function of strain strength. Gauge factor $(G F)$ is defined from the relation: $\Delta I / I(\mathrm{ppm})=G F$. strain (ppm), where $I$ is the optical emission intensity.

Surface adsorption of molecular species in nanofibers assembled from suitably functionalized oligomers (Schiek et al., 2005) could generate photonic chemosensing, e.g., by modulation of the effective refractive index of the nanofiber resonance modes. Photonic sensitivity could be further enhanced near lasing threshold, as demonstrated in recent reports (Rose et al., 2005; Chen et al., 2011).

\section{Heteroepitaxial nanofibers based on the $p-6 \mathrm{P} / 6 \mathrm{~T}$ system}

Tuning of lasing wavelength of $p-6 \mathrm{P}$ epitaxial nanofibers can possibly be achieved by crystal doping with long-wavelength emitting molecules. With this aim we study the growth and optical properties of $p-6 \mathrm{P} /$ sexithiophene (6T) heteroepitaxial films by hot-wall epitaxy on (001)-oriented muscovite mica. Both $p-6 \mathrm{P}$ and $6 \mathrm{~T}$ are good model systems for heteroepitaxy and important oligomers for optoelectronic device applications. It has been demonstrated that both $p$-6P and $6 \mathrm{~T}$ crystalline thin films can be used as organic templates to obtain highly crystalline organic-organic heterostructures in which $p-6 \mathrm{P}$ and $6 \mathrm{~T}$ molecules are both standing or parallel to the substrate (Oehzelt et al., 2006; Koller et al., 2006). We show by hot-wall heteroepitaxy that $p$-6P nanofiber templates grown on muscovite mica can be used to obtain oriented and mutually parallel $6 \mathrm{~T} / p$-6P nanofibers whose emission color depends on the relative concentrations of the two components. We resort to a growth chamber equipped with two hot-wall epitaxy reactors for serial deposition of $p-6 \mathrm{P}$ and $6 \mathrm{~T}$. The substrate is first exposed to the reactor with the $p$-6P source and than transferred to the $6 \mathrm{~T}$ reactor; details can be found in (Simbrunner, 2010). 

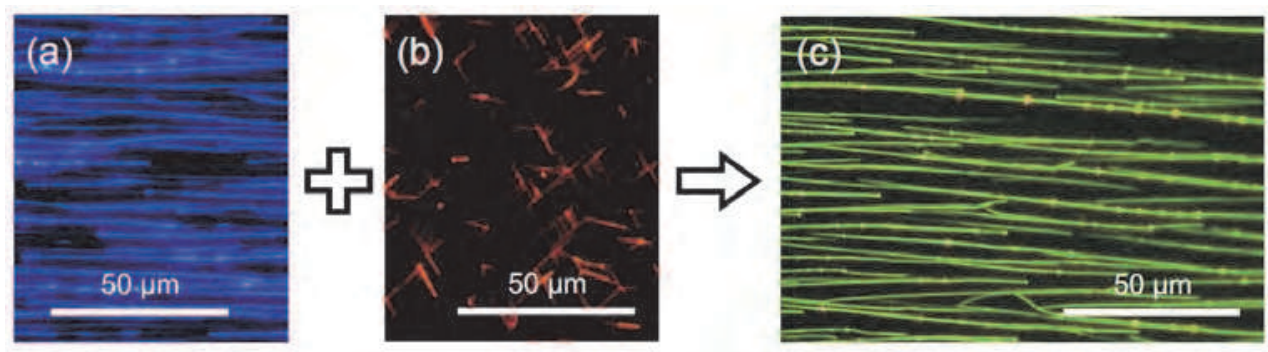

Fig. 11. Fluorescence micrographs of heteroepitaxial nanofibers grown on (001)-oriented muscovite mica by hot-wall epitaxy at $120^{\circ} \mathrm{C}$ substrate temperature: (a) p-6P; (b) 6T; (c) bilayer of 6T (deposition time $90 \mathrm{~min}$.) on $p$-6P (deposition time $40 \mathrm{~min}$.). Epifluorescence is excited by UV lamp irradiation.

When $6 \mathrm{~T}$ is directly grown on muscovite, short nanofibers with red-orange fluorescence are yielded with several orientations, whereas 6T deposition on a template of $p-6 \mathrm{~T}$ nanofibers results in $6 \mathrm{~T} / p$-6P bilayer fibers which maintain the fiber template's orientation, with no $6 \mathrm{~T}$ material being deposited in between adjacent p-6P fibers. This is clearly demonstrated by the epifluorescence micrographs shown in Fig. 11. Green emitting fibers are obtained with a 6T deposition time of $5 \mathrm{~s}$, corresponding to submonolayer coverage. In this limit, the 6T fluorescence spectrum is similar to that of a solvated 6T phase (green line spectrum labeled as 6T(i) in Fig. 12).

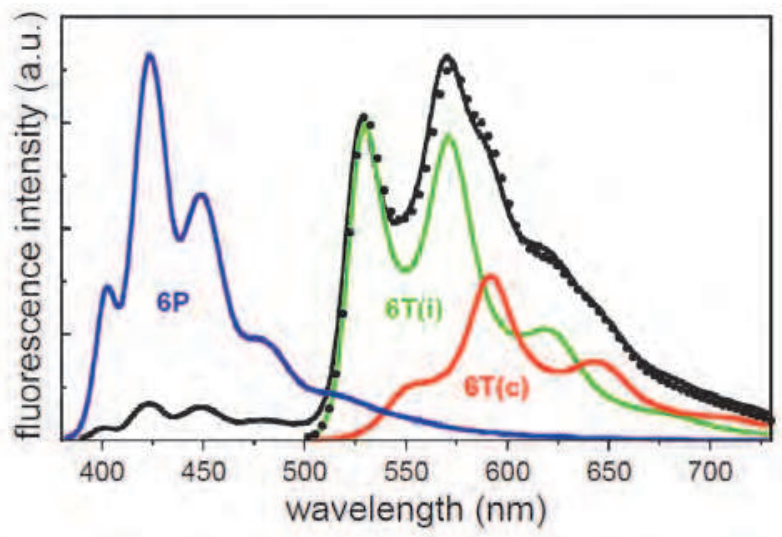

Fig. 12. Fluorescence spectra of pure $p$-6P nanofibers (blue line) and $6 \mathrm{~T} / p-6 \mathrm{P}$ bilayer nanofibers (black line). Fluorescence is excited by $370 \mathrm{~nm}$ laser pulses at $80 \mathrm{MHz}$ repetition rate. Green and red spectra belong to interfacial (i) and bulk crystal (c) $6 \mathrm{~T}$ in the bilayer sample. Black dots are the sum of 6T(i) and 6T(c) spectra.

Increasing 6T deposition time (and so the 6T-to-6P concentration ratio), oriented 6T crystalline fibers are deposited on top of $p-6 \mathrm{P}$ templating fibers. Crystal phase emission, as obtained in pure 6T films, is shown as the red line labeled as 6T(c) in Fig. 12. The overall emission spectrum of the $6 \mathrm{~T} / p$-6P bilayer nanofiber film grown with $40 \mathrm{~min}$. $(p-6 \mathrm{P})$ and 90 
min. (6T) deposition times (black line in Fig. 12) shows evidence of strong quenching of the $p$-6P emission. The interfacial, green emitting $6 \mathrm{~T}$ sheet is highly emissive, its integrated intensity being comparable to that of the $\sim 400-\mathrm{nm}$-thick $6 \mathrm{~T}$ crystalline overlayer. The emission strengths of the interfacial and crystal components are determined by fitting a linear combination of the spectra of the two 6T phases to the total emission spectrum.

Quenching of $p-6 \mathrm{P}$ emission is ascribed to efficient sensitization of 6T via resonance energy transfer (Wieb Van Der Meer et al., 1994). The $p$-6P/6T material couple is thus inferred to display type-I alignment of electronic levels in the bilayer structure, which could allow for extending operation of $p-6 \mathrm{P}$ epitaxial nanofiber lasers to $6 \mathrm{~T}$ emission wavelengths in the green and in the red via resonance energy transfer from $p$-6P to 6T.
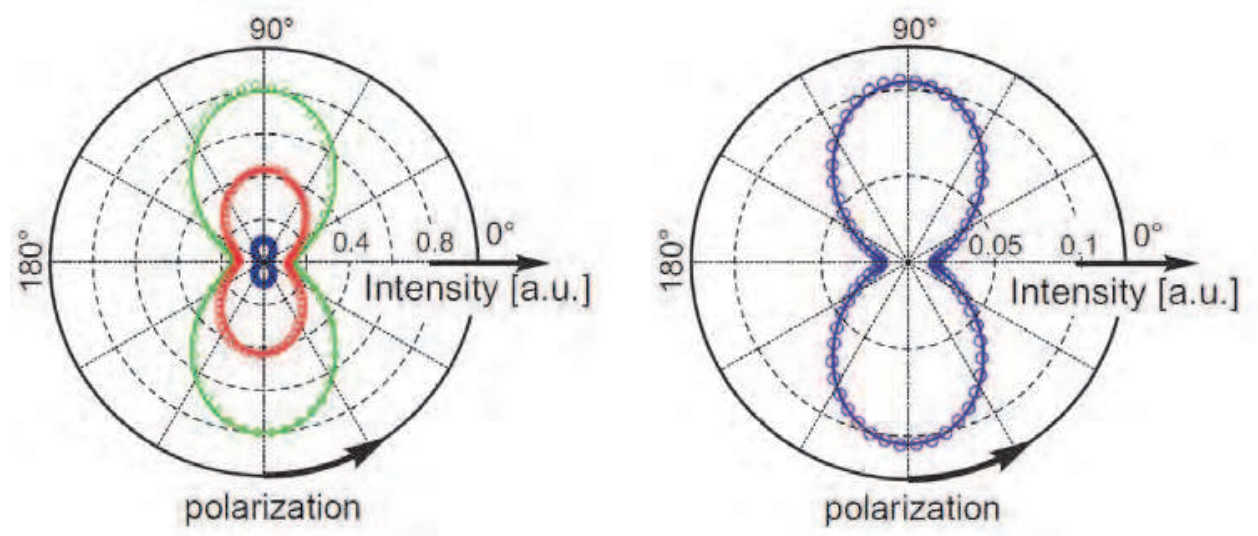

Fig. 13. Polar plots of the emission intensity of $6 \mathrm{~T} / p-6 \mathrm{P}$ bilayer film versus analyzer polarizaton angle. Dots: experimental points; lines: fits of Malus' law function to the experimental data. Blue, green and red colors code for spectrally-integrated emission intensity of $p-6 \mathrm{P}, 6 \mathrm{~T}(\mathrm{i})$ and $6 \mathrm{~T}(\mathrm{c})$ material phases, respectively. A magnified view of $p-6 \mathrm{P}$ intensity data is shown in the right panel for better visibility.

Long-range azimuthal order is demonstrated in $6 \mathrm{~T} / p-6 \mathrm{P}$ bilayer nanofibers by polarizationresolved fluorescence intensity measurements. While the pump laser polarization is set parallel to the $p-6 \mathrm{P}$ long molecular axis for maximum absorption, the collected fluorescence signal is analyzed by a rotating linear polarized. For each angular position of the polarizer, the emission spectrum is acquired and decomposed in its $p$-6P (blue), interfacial 6T (green) and crystalline 6T (red) components. The integrated intensities of the three materials' components are then plotted versus polarizer rotation angle. The results fit quite well to Malus' $\left(\sin ^{2}\right)$ law dependences for all the three materials' phases. In particular, optical emission dipoles of $p-6 \mathrm{P}$ and $6 \mathrm{~T}$ are found to be parallel over domains as large in area as $\sim 1$ $\mathrm{cm}^{2}$. These optical properties support the results X-ray diffraction studies, which determine the exact epitaxial relationships between the $6 \mathrm{~T} / p-6 \mathrm{P}$ crystal phases and muscovite mica substrate (Simbrunner et al., 2010). Despite the potential exhibited by $6 \mathrm{~T} / p-6 \mathrm{P}$ heteroepitaxial fibers on oriented muscovite mica for broadband laser operation, at the time this review is being written no evidence of $6 \mathrm{~T}$ lasing action in such nanostructures or in other epitaxial thin films has been reported yet. 


\section{Conclusion}

We reviewed the photonic and photophysical properties of $p$-6P epitaxial nanofibers grown by hot-wall epitaxy and organic molecular beam epitaxy on (001)-oriented muscovite mica. We presented experimental data on amplified spontaneous emission and coherent random lasing in ensembles of nanofibers, as well as in singly selected fibers, where waveguide amplification performance and coherent feedback mechanisms were found out. We also reported the excited-state nonlinear dynamics of nanofibers, which was studied by transient photoluminescence and absorption spectroscopy, and presented a quantitative account of the main photophysical processes occurring in photoexcited nanofibers, namely, stimulated emission and excited-state absorption. After a brief discussion on potential applications of organic epitaxial nanofibers in photonic sensing, we concluded by showing recent results obtained on mixed $6 \mathrm{~T} / p-6 \mathrm{P}$ heteroepitaxial nanofibers. The $6 \mathrm{~T} / \mathrm{p}-6 \mathrm{P}$ material system, combined with the potential of organic epitaxy techniques, looks promising for the realization of nanoscale laser devices operating in the blue, green and red spectral regions.

\section{Acknowledgment}

We acknowledge the scientific collaboration of F. Cordella, F. Floris, M. Marceddu, R. Orrù, A. Andreev, G. Hernandez-Sosa, N. S. Sariciftci, G. Schwabegger, C. Simbrunner, H. Sitter, M. Oehzelt, R. Resel, F. Balzer, V.G. Bordo and H.-G. Rubahn.

\section{References}

Andreev, A.; Matt, G.; Brabec, C. J.; Sitter, H.; Badt, D.; Seyringer, H. \& Sariciftci, N. S. (2000). Highly Anisotropically Self-Assembled Structures of para-Sexiphenyl Grown by Hot-Wall Epitaxy. Adanced Materials, Vol. 12, No. 9, (May 2000), pp. 629633, ISSN 0935-9648

Andreev, A., Sitter, H., Brabec, C. J.; Hinterdorfer, P.; Springholz, G. \& Sariciftci, N. S. (2001). Self-assembled growth of highly oriented para-sexiphenyl thin films. Synthetic Metals, Vol. 121, No. 1-3, (March 2001), pp. 1379-1380, ISSN 0379-6779

Andreev, A.; Quochi, F.; Cordella, F.; Mura, A.; Bongiovanni, G.; Sitter, H.; Hlawacek, G.; Teichert, C. \& Sariciftci, N. S. (2006). Coherent random lasing in the deep blue from self-assembled organic nanofibers. Journal of Applied Physics, Vol. 99, No. 3, (February 2006), pp. 034305(1-6), ISSN 0021-8979

Balzer, F. \& Rubahn, H.-G. (2001). Dipole-assisted self-assembly of light-emitting $p$-nP needles on mica. Applied Physics Letters, Vol. 79, No. 23, (December 2001), pp. 38603862, ISSN 0003-6951

Balzer, F.; Bordo, V. G.; Simonsen, A. C. \& Rubahn, H.-G. (2003). Optical waveguiding in individual nanometer-scale organic fibers. Physical Review B, Vol. 67, No. 11, (March 2003), pp. 115408(1-8), ISSN 1098-0121

Birendra-Singh, T.; Hernandez-Sosa, G.; Neugebauer, H.; Andreev, A.; Sitter, H. \& Sariciftci, N. S. (2006). Electrical transport properties of hot wall epitaxially grown parasexiphenyl nano-needles. physica status solidi (b), Vol. 243, No. 13, (November 2006), pp. 3329-3332, ISSN 0370-1972

Cao, H. (2003). Lasing in random media. Waves in Random and Complex Media, Vol. 13, No. 3, (? 2003), pp. R1-R39, ISSN 1745-5030 
Cao, H. (2005). Review on latest developments in random lasers with coherent feedback. Journal of Physics A: Mathematical and General, Vol. 38, No. 49, (December 2005), pp. 10497-10535, ISSN 0305-4470

Chen, R.; Ling, B.; Sun, X. W. \& Sun, H. D. (2011). Room Temperature Excitonic Whispering Gallery Mode Lasing from High-Quality Hexagonal ZnO Microdisks. Advanced Materials, Vol. xx, No. Xx, (published online April 2011), DOI: 10.1002/adma.201100423, ISSN 0935-9648

Cordella, F.; Quochi, F.; Saba, M.; Andreev, A.; Sitter, H., Sariciftci, N. S.; Mura, A. \& Bongiovanni, B. (2007). Optical Gain Performance of Epitaxially Grown paraSexiphenyl Films. Advanced Materials, Vol. 19, No. 17, (September 2007), pp. 22522256, ISSN 0935-9648

Kadashchuk, A.; Andreev, A.; Sitter, H.; Sariciftci, N. S.; Skryshevsky, Y.; Piryatinski, Y.; Blonsky, I. \& Meissner, D. (2004). Aggregate States and Energetic Disorder in Highly Ordered Nanostructures of para-Sexiphenyl Grown by Hot Wall Epitaxy. Advanced Functional Materials, Vol. 14, No. 10, (October 2004), pp. 970-978, ISSN 1616-301X

Kankate, L.; Balzer, F.; Niehus, H. \& Rubahn, H.-G. (2008). From clusters to fibers: Parameters for discontinuous para-hexaphenylene thin film growth. Journal of Chemical Physics, Vol. 128, No. 8, (February 2008), pp. 084709(1-12), ISSN 0021-9606

Kjelstrup-Hansen, J.; Henrichsen, H. H.; Bøggild, P. \& Rubahn, H.-G. (2006). Electrical properties of a single $p$-hexaphenylene nanofiber. Thin Solid Films, Vol. 515, No. 2, (October 2006), pp. 827-830, ISSN 0040-6090

Koller, G.; Berkebile, S.; Krenn, J. R.; Netzer, F. P.; Oehzelt, M.; Haber, T.; Resel, R. \& Ramsey, M. G. (2006). Heteroepitaxy of Organic-Organic Nanostructures. Nano Letters, Vol. 6, No. 6, (June 2006), pp. 1207-1212, ISSN 1530-6984

Oehzelt, M.; Koller, G.; Ivanco, J.; Berkebile, S.; Haber, T.; Resel, R.; Netzer, F. P. \& Ramsey, M. G. Organic (2006). Heteroepitaxy: $p$-Sexiphenyl on Uniaxially Oriented $\alpha-$ Sexithiophene. Advanced Materials, Vol. 18, No. 18, (September 2006), pp. 2466-2470, ISSN 0935-9648

Plank, H.; Resel, R.; Purger, S.; Keckes, J.; Thierry, A.; Lotz, B.; Andreev, A.; Sariciftci, N. S. \& Sitter, H. (2001). Heteroepitaxial growth of self-assembled highly ordered parasexiphenyl films: A crystallographic study. Physical Review B, Vol. 64, No. 23, (December 2001), pp. 235423(1-5), ISSN 1098-0121

Plank, H.; Resel, R.; Sitter, H.; Andreev, A.; Sariciftci, N. S.; Hlawacek, G.; Teichert, C.; Thierry, A. \& Lotz, B. (2003). Molecular alignments in sexiphenyl thin films epitaxially grown on muscovite. Thin Solid Films, Vol. 443, No. 1-2, (October 2003), pp. 108-114, ISSN 0040-6090

Quochi, F.; Cordella, F.; Orrù, R.; Communal, J. E.; Verzeroli, P.; Mura, A.; Bongiovanni, G.; Andreev, A.; Sitter, H. \& Sariciftci, N. S. (2004). Random laser action in selforganized para-sexiphenyl nanofibers grown by hot-wall epitaxy. Applied Physics Letters, Vol. 84, No. 22, (May 2004), pp. 4454-4456, ISSN 0003-6951

Quochi, F.; Cordella, F.; Mura, A.; Bongiovanni, G.; Balzer, F. \& Rubahn, H.-G. (2005). OneDimensional Random Lasing in a Single Organic Nanofiber. The Journal of Physical Chemistry B, Vol. 109, No. 46, (November 2005), pp. 21690-21693, ISSN 1520-6106 
Quochi, F.; Cordella, F.; Mura, A.; Bongiovanni, G.; Balzer, F. \& Rubahn, H.-G. (2006). Gain amplification and lasing properties of individual organic nanofibers. Applied Physics Letters, Vol. 88, No. 4, (January 2006), pp. 041106(1-3), ISSN 0003-6951

Quochi, F.; Saba, M.; Cordella, F.; Gocalinska, A.; Corpino, R.; Marceddu, M.; Anedda, A.; Andreev, A.; Sitter, H.; Sariciftci, N. S.; Mura, A. \& Bongiovanni, G. (2008). Temperature Tuning of Nonlinear Exciton Processes in Self-Assembled Oligophenyl Nanofibers under Laser Action. Advanced Materials, Vol. 20, No. 16, (August 2008), pp. 3017-3021, ISSN 0935-9648

Rose, A.; Zhu, Z.; Madigan, C. F.; Swager, T. M. And Bulović, V. (2005). Sensitivity gains in chemosensing by lasing action in organic polymers. Nature, Vol. 434, No. 7035, (April 2005), pp. 876-879, ISSN 0028-0836

Schiek, M.; Lützen, A.; Koch, R.; Al-Shamery, K.; Balzer, F.; Frese, R. \& Rubahn, H.-G. (2005). Nanofibers from functionalized para-phenylene molecules. Applied Physics Letters, Vol. 86, No. 15, (Dec 2005), pp. 153107(1- 3), ISSN 0003-6951

Simbrunner, C.; Quochi, F.; Hernandez-Sosa, G.; Oehzelt, M.; Resel, R.; Hesser, G.; Arndt, M.; Saba, M.; Mura, A.; Bongiovanni, G. \& Sitter, H. (2010). Organic-Organic Heteroepitaxy of Red-, Green-, and Blue-Emitting Nanofibers, ACSNano, Vol. 4, No. 10, (October 2010), pp. 6244-6250, ISSN 1936-0851

Simbrunner, C.; Nabok, D.; Hernandez-Sosa, G.; Oehzelt, M.; Djuric, T.; Resel, R.; Romaner, L.; Puschnig, P.; Ambrosch-Draxl, C.; Salzmann, I.; Schwabegger, G.; Watzinger, I. \& Sitter, H. (2011). Epitaxy of Rodlike Organic Molecules on Sheet Silicates - A Growth Model Based on Experiments and Simulations, Journal of The American Chemical Society, Vol. 133, No. 9, (March 2011), pp. 3056-3062, ISSN 0002-7863

Stampfl, J.; Tasch, S.; Leising, G. \& Sherf, U. (1995). Quantum Efficiencies of Electroluminescent Poly(para-phenylenes). Synthetic Metals, Vol. 71, No. 1-3, (April 1995), pp. 2125-2128, ISSN 0379-6779

Wieb Van Der Meer, B.; Coker, G. III \& Simon Chen, S.-Y. (August 9, 1994). Resonance Energy Transfer: heory and Data (1 ${ }^{\text {st }}$ edition), John Wiley \& Sons, ISBN 0471185892, New York

Wiersma, D. S. (2000). The smallest random laser. Nature, Vol. 406, No. 6792, (July 2000), pp. 132-133, ISSN 0028-0836

Wiersma, D. S. \& Cavalieri, S. (2001). Light emission: A temperature-tunable random laser. Nature, Vol. 414, No. 6865, (December 2001), pp. 708-709, ISSN 0028-0836

Wiesenhofer, H.; Zojer, E.; List, E. J. W.; Scherf, U.; Brédas, J.-L. \& Beljonne, D. (2006). Molecular Origin of the Temperature-Dependent Energy Migration in a Rigid-Rod Ladder-Phenylene Molecular Host. Advanced Materials, Vol. 18, No. 3, (February 2006), pp. 310-314, ISSN 0935-9648

Yanagi, H. \& Morikawa, T. (1999). Self-waveguided blue light emission in $p$-sexiphenyl crystals epitaxially grown by mask-shadowing vapor deposition. Applied Physics Letters, Vol. 75, No. 2, (July 1999), pp. 187-189, ISSN 0003-6951 


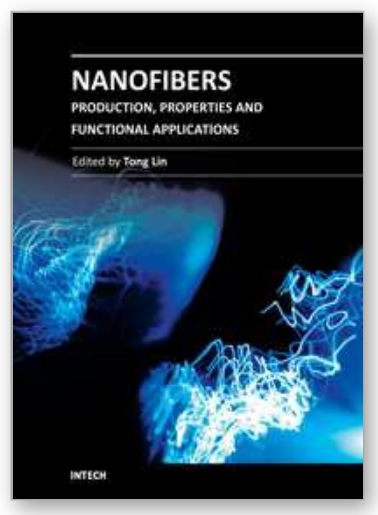

\author{
Nanofibers - Production, Properties and Functional Applications \\ Edited by Dr. Tong Lin
}

ISBN 978-953-307-420-7

Hard cover, 458 pages

Publisher InTech

Published online 14, November, 2011

Published in print edition November, 2011

As an important one-dimensional nanomaterial, nanofibers have extremely high specific surface area because of their small diameters, and nanofiber membranes are highly porous with excellent pore interconnectivity. These unique characteristics plus the functionalities from the materials themselves impart nanofibers with a number of novel properties for advanced applications. This book is a compilation of contributions made by experts who specialize in nanofibers. It provides an up-to-date coverage of in nanofiber preparation, properties and functional applications. I am deeply appreciative of all the authors and have no doubt that their contribution will be a useful resource for anyone associated with the discipline of nanofibers.

\title{
How to reference
}

In order to correctly reference this scholarly work, feel free to copy and paste the following:

Francesco Quochi, Michele Saba, Andrea Mura and Giovanni Bongiovanni (2011). Photophysics and Photonics of Heteroepitaxial Organic Nanofibers, Nanofibers - Production, Properties and Functional Applications, Dr. Tong Lin (Ed.), ISBN: 978-953-307-420-7, InTech, Available from:

http://www.intechopen.com/books/nanofibers-production-properties-and-functional-applications/photophysicsand-photonics-of-heteroepitaxial-organic-nanofibers

\section{INTECH}

open science | open minds

\section{InTech Europe}

University Campus STeP Ri

Slavka Krautzeka 83/A

51000 Rijeka, Croatia

Phone: +385 (51) 770447

Fax: +385 (51) 686166

www.intechopen.com

\section{InTech China}

Unit 405, Office Block, Hotel Equatorial Shanghai

No.65, Yan An Road (West), Shanghai, 200040, China

中国上海市延安西路65号上海国际贵都大饭店办公楼405单元

Phone: +86-21-62489820

Fax: +86-21-62489821 
(C) 2011 The Author(s). Licensee IntechOpen. This is an open access article distributed under the terms of the Creative Commons Attribution 3.0 License, which permits unrestricted use, distribution, and reproduction in any medium, provided the original work is properly cited. 